\title{
Perfecting the separation and measurement of calixarene Dioctyl Calix-[4]-Crown- 6 by high performance liquid chromatography with UV detection
}

\author{
A. Dodi \\ Direction du Cycle du Combustible - Département d'Entreposage et de Stockage des Déchets, C.E.A. Cadarache, \\ 13108 Saint-Paul-lez-Durance, France
}

\begin{abstract}
Calix-[4]-crown-6 display an exceptional selectivity towards cesium, due to their preorganization and their complementarity between the size of the crown and that of this cation. Their application in the field of nuclear industry allows this element to be very selectively extracted from nitric solutions obtained from the dissolution of nuclear spent fuel. The studies dealing with extraction of cesium by calixarenes involve knowing how to measure this complexing molecule into its solvent. The solvent is a mixture of Nitro-2-PhenylHexylEther (NPHE) and Tetra Propylene Hydrogenated (TPH). Since TPH is saturated, and thus, non UV absorbing molecule, its presence into the mixture will not interfere; on the other hand, NPHE, which exhibits chemical fonctionalities and an hydrophobicity quite similar to those of calixarene, must be separated from the complexing molecule. We have determined the conditions of separation between calixarene and its solvent by using the adsorption chromatographic mode over silica, associated with an organic eluent composed of hexane and isopropanol. In these conditions, separation of the calixarene from NPHE is accomplished in less than 8 minutes.
\end{abstract}

Keywords. Calixarene - adsorption chromatography.

\section{Introduction}

Today, the nuclear industry needs to separate the waste it generates in order to optimize its management. Another possibility consists in separating some long lived radionuclides for the purpose of their subsequent transmutation into short lived or stable isotopes. Cesium 135 is one of the fission products for which this kind of transformation is envisioned. The separation of this element from the fission solutions arising from reprocessing operations has already been studied [1-4]. The more promising studies consists in extraction of cesium from an aqueous nitric solution using a calixarene molecule diluted into a solvent composed of a $\mathrm{C}_{12}$ branched saturated alkane: Tetra Propylene.

Hydrogenated (TPH) and a nitrated aromatic ether: nitro2-PhenylHexylEther (NPHE). The complexant molecule is dissolved at about a few grams per liter into this solvent, then the liquid/liquid extraction of cesium from the aqueous nitric solution is performed. The neutral Calixarene-Cs complex is recovered in the organic phase.

$$
\mathrm{Cs}^{+}{ }_{\mathrm{aq}}+\mathrm{NO}_{3}{ }_{\text {aq }}^{-}+\text {Calixarene }_{\text {org }} \Leftrightarrow\left(\text { CalixareneCs }{ }^{+} \mathrm{NO}_{3}{ }^{-}\right)_{\text {org }}
$$

The chromatographic separation of calixarene from the species TPH and NPHE is required in order to perform measurement. Saturated alcanes (TPH) do not absorb UV light, so the main difficulty lies in separating calixarene from
NPHE which exhibits about the same chemical functions as the calixarene molecule.

To our knowledge, at present no method has been developed to perform this measurement.

\section{Experimental}

\section{Instrumentation}

A 1100 Hewlett Packard HPLC system equipped with a quaternary gradient pump, an on-line degassing system, an automatic sampler and a diode array detector was used. The DAD detector allows spectral characterization of the eluted compounds between 200 and $800 \mathrm{~nm}$.

The thin layer chromatography experiments were performed using a Merck silica gel 60F254 placed on a glass plate $(5 \times 20 \mathrm{~cm})$.

\section{Reagents}

Tetra Propylene Hydrogenated and Nitro-2-PhenylHexylEther (NPHE) were analytical grade products.

Hexane was purchased from Merck (minimal purity: $99 \%$ and max. water content: $0.01 \%$ )

2-Propanol was purchased from Prolabo (minimal purity: $99 \%$ and max. water content: $0.5 \%$ ). 


\section{Original articles}

\section{The implementation of High Performance Liquid Chromatography}

The technique we have decided to use is the HPLC associated with a UV detection mode. In the process, it is necessary to have a technique with an implementation that does not require the use of overly sophisticated material (for example: mass spectrometer).

Two chromatographic modes have been tested:

- partition chromatography;

- adsorption chromatography.

\section{Partition chromatography}

Partition chromatography was performed with a $5 \mu \mathrm{m}$ particle $\mathrm{C}_{18}$ Nucleosil $(250 \times 4.6)$. We have tested this phase in association with mobile phases composed of different water/methanol or water/acetonitrile mixtures $(0 \leq$ water percent $\leq 10)$. The corresponding chromatograms show a peak for the NPHE molecule but the calixarene does not elute from the chromatographic system. This may be explained by the presence of two apolar crowns in the structure of calixarene (oxygenated crown and tetraaryl cavity; see figure 1). It can be assumed that octadecyl chains wrap around the apolar crowns of calixarene maintaining the complexing agent permanently attached to the stationary phase.

\section{Adsorption liquid chromatography}

On the other hand, adsorption chromatography uses a very polar stationary phase so interactions with a very apolar molecule, such as calixarene, are considerably reduced.

An initial approach using the Thin Layer chromatography (TLC) has allowed us to develop an eluent mixture adapted for the separation of the two molecules (calixarene and NPHE)

This eluent is composed of a mixture of $85 \%$ hexane and $15 \%$ 2-propanol. In these mobile phase conditions, and with a wavelength of $254 \mathrm{~nm}$ we observe a spot at the solvent top line (NPHE) and another spot characterized by a Rf of about 0.25 .

In order to optimize the detection of the calixarene molecule, we have recorded the UV spectrum of calixarene and NPHE. These spectra are reported in figures 2 and 3.

The optimized conditions, deduced from T.L.C. approach and UV spectra are:

Stationary phase:

- Silica Uptisphere with $5.0 \mu \mathrm{m}$ particle size

Mobile phase:

- Hexane $85 \%$

- 2-Propanol $15 \%$

- Elution speed: $1.2 \mathrm{ml} / \mathrm{minute}$

Detection wavelength: $228 \mathrm{~nm}($ Bandwith $=16 \mathrm{~nm})$
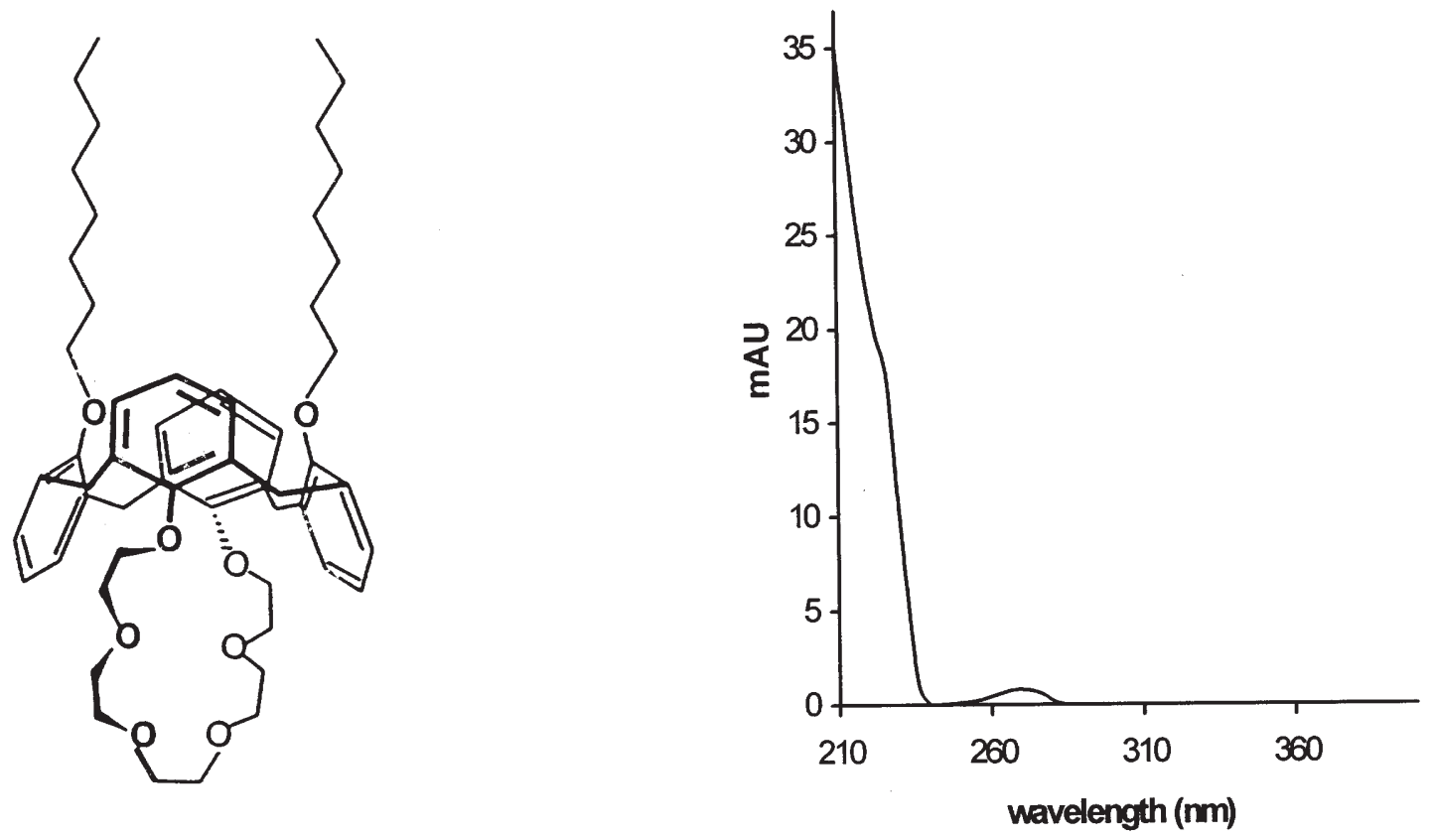

Figure 1. Structure of Calixarene Dioctyl Calix-[4]-Crown-6.

Figure 2. UV Spectrum of Calixarene. 


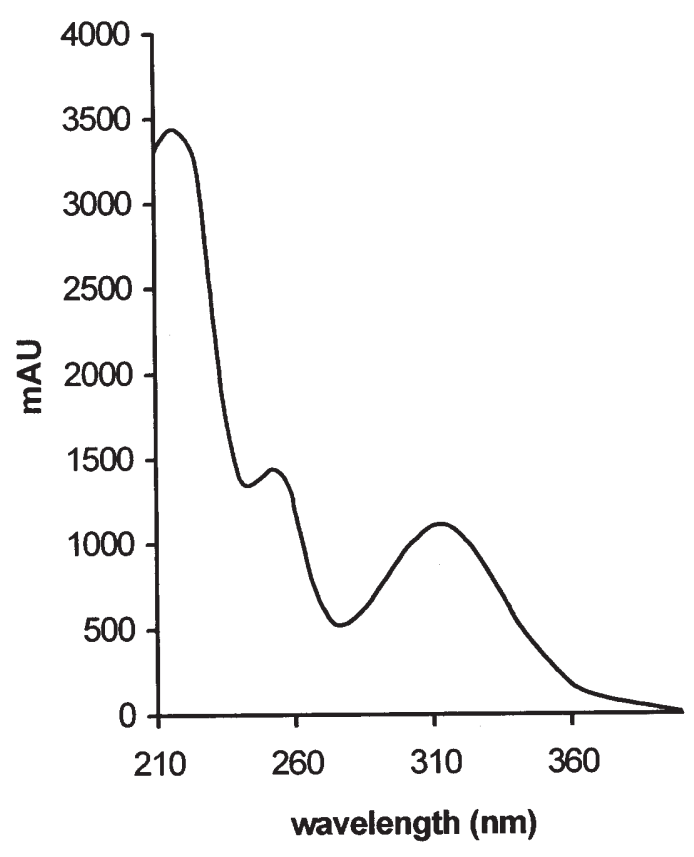

Figure 3. UV Spectrum of NPHE.

With such conditions the chromatogram of figure 4 can be obtained:

Injected quantity: $5.0 \mu \mathrm{l}$ of a $5 \mathrm{~g} / \mathrm{l}$ solution of calixarene in the mixture TPH/NPHE previously diluted 200 times into hexane. (This corresponds to an effective concentration of $25 \mathrm{mg} / \mathrm{l}$ calixarene into hexane).

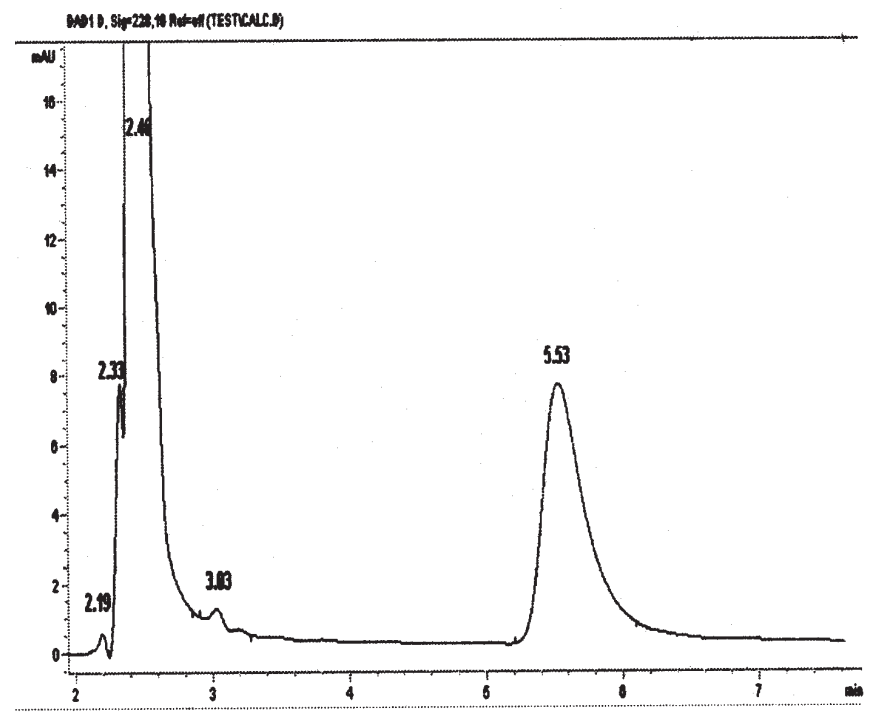

Figure 4. Separation between calixarene and NPHE.
Interpretation: 2.46 min: NPHE; 5.53 min: Dioctyl Calix[4]crown-6. The two low intensity peaks eluted in front of the NPHE peak certainly correspond to the two other position isomers of NPHE.

Using different concentration solutions of calixarene, the following calibration curve is obtained:

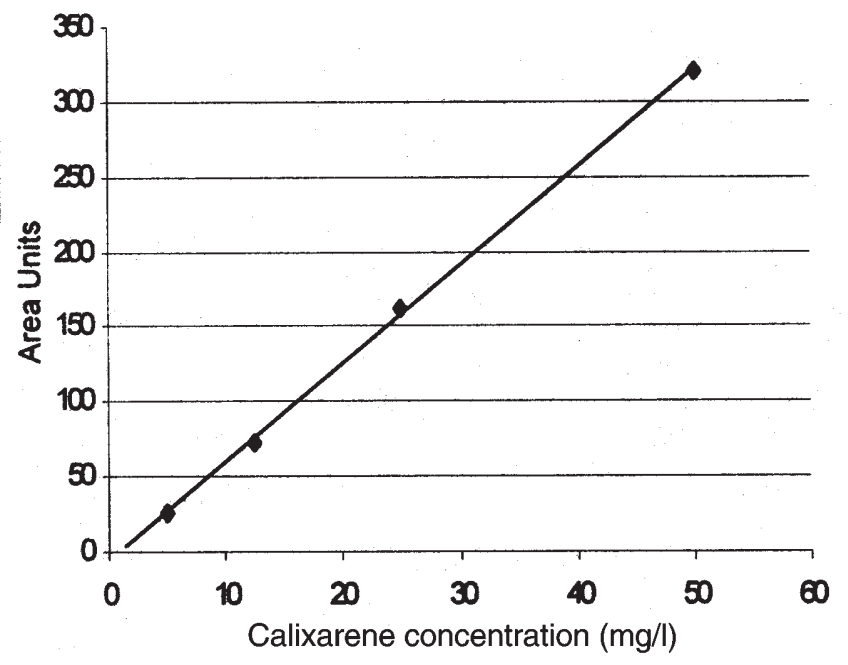

Figure 5. Calibration curve of calixarene diluted into hexane.

Correlation coefficient $=0.99955$.

Slope $=6.496$.

Origin value $=-3.878$.

A statistical study has been carried out with two solutions of calixarene and NPHE into hexane (Tab. I).

We have observed that the use of an area peak allows better repeatability than peak height. Thus, a $12.5 \mathrm{mg} / \mathrm{l}$ Calixarene solution leads to an uncertainty value of $4.2 \%$ if heights are considered and $2.7 \%$ if areas are used.

The detection limit estimated from the signal/noise ratio is about $2 \mathrm{mg} / \mathrm{l}$. The previous repeatability study has allowed us to calculate the standard deviation repeatability. If we consider that the detection limit is 3 times the value of the standard deviation, we obtain an area value which represents this limit. This value, integrated into the equation of the calibration curve, leads to a calculated detection limit of $1.85 \mathrm{mg} / \mathrm{l}$, i.e. $1.85 \times 200=370 \mathrm{mg} / \mathrm{l}$ in the initial undiluted solution.

\section{Conclusion}

The separation and measurement of calixarene Dioctyl Calix-[4]-Crown-6 diluted into a mixture TPH/NPHE (70/30 


\section{Original articles}

Table I.

\begin{tabular}{lcccc}
\hline Calixarene/NPHE & \multicolumn{2}{c}{$12.5 \mathrm{mg} / \mathrm{l}$} & \multicolumn{2}{c}{$25 \mathrm{mg} / \mathrm{l}$} \\
& height & area & height & area \\
\hline 1 & 3.42 & 70.4 & 8.84 & 155.8 \\
2 & 3.08 & 68.7 & 9.04 & 163.8 \\
3 & 3.38 & 71.3 & 8.07 & 160.9 \\
4 & 3.36 & 70.7 & 7.51 & 165.5 \\
5 & 3.01 & 73.6 & 7.42 & 161.9 \\
6 & 2.92 & 71.6 & 7.47 & 167.0 \\
7 & 3.23 & 72.6 & - & - \\
8 & 3.24 & 73.3 & - & - \\
9 & 3.48 & 76.4 & - & - \\
10 & 3.41 & 77.5 & - & - \\
Mean & 3.25 & 72.6 & 8.06 & 162.5 \\
$\sigma$ & 0.19 & 2.72 & 0.73 & 3.97 \\
\multicolumn{1}{c}{$\sigma \cdot t_{0.05}$} & & & & \\
Mean. $\sqrt{n}$ & 0.042 & 0.027 & 0.094 & 0.026 \\
\hline
\end{tabular}

- v/v), which represents the mixture used in the cesium extraction process, is achieved in less than 8 minutes. NPHE is approximately eluted in the dead volume of the chromatographic column, when calixarene is eluted in a volume about 2.5 times greater. The detection limit is about $370 \mathrm{mg}$ of calixarene per liter of sample solution; if necessary this limit may be decreased by injecting higher volumes. Another solution would consist in the realization of an elution gradient allowing the elution of NPHE and the retention of calixarene over the stationary phase (for example, with a mobile phase only composed of hexane); afterwards, an increase of mobile phase polarity, by increasing the level of 2-propanol, will allow the elution of calixarene.

A repeatability study has shown that it is more interesting to consider area peaks rather than height peaks. A relative uncertainty of about $3 \%$ in the middle range of the calibration curve is obtained, for a confidence interval of $95 \%$.

It would be interesting to extend this study to other calixarenes (with similar polarity) in order to see if this method allows the characterization (i.e. a differential elution) of the different molecules presently available and which might lead to an industrial application.

\section{References}

1. Ungaro, R., Casnati, A.; Ugozzoli, F.; Pochini, A.; Dozol, J.F.; Hill, C.; Rouquette, H. Angew. Chem. Int. Ed. Engl. 1994, 33, $\mathrm{n}^{\circ} 14,1506-1509$.

2. Hill, C.; Dozol, J.F.; Lamare, V.; Rouquette, H.; Eymard, S.; Tournois, B.; Vicens, J.; Asfari, Z.; Bressot C.; Ungaro, R.; Casnati, A. J. Incl. Phenom. 1994, 19, 399-408.

3. Dozol, J.F.; Simon, N.; Rouquette, H.; Eymard, S.; Tournois, B.; Lamare, V.; Lecomte, M.; Masson, M.; Viallesoubranne, C. Gobal 95, 1995, Versailles, France.

4. Hill, C.; Dozol, J.F.; Rouquette, H.; Eymard, S.; Tournois, B. J. Membrane Sci. 1996, 114, 73-80. 